\title{
Clinical Study \\ Effects of Marine Phospholipids Extract on the Lipid Levels of Metastatic and Nonmetastatic Prostate Cancer Patients
}

\author{
Daniela Küllenberg de Gaudry, ${ }^{1,2}$ Lenka A. Taylor, ${ }^{3}$ Jessica Kluth, ${ }^{1}$ Tobias Hübschle, ${ }^{4}$ \\ Jonas Fritzsche, ${ }^{4}$ Bernd Hildenbrand, ${ }^{1}$ Lars Pletschen, ${ }^{1}$ Karin Schilli, ${ }^{5}$ Arwen Hodina, \\ Lee S. Griffith, ${ }^{6}$ Jürgen Breul, ${ }^{4}$ Clemens Unger, ${ }^{7}$ and Ulrich Massing ${ }^{1,8}$
}

${ }^{1}$ Department of Lipids \& Liposomes, Tumor Biology Center, Breisacher Straße 117, 79106 Freiburg, Germany

${ }^{2}$ Hermann Staudinger Graduate School, the University of Freiburg, Hebelstraße 27, 79104 Freiburg, Germany

${ }^{3}$ Pharmacy Department, University Hospital Heidelberg, Im Neuenheimer Feld 670, 69126 Heidelberg, Germany

${ }^{4}$ Loretto Hospital, Mercystraße 6-14, 79100 Freiburg, Germany

${ }^{5}$ Urological practice U3, Bertoldstraße 45, 79098 Freiburg, Germany

${ }^{6}$ PgXpertise, Bahnhofstraße 8, 79288 Gottenheim, Germany

${ }^{7}$ Center for Cancer Medicine, Breisacher Straße 84 B, 79110 Freiburg, Germany

${ }^{8}$ Department of Clinical Research, Tumor Biology Center, Breisacher Straße 117, 79106 Freiburg, Germany

Correspondence should be addressed to Ulrich Massing; massing@tumorbio.uni-freiburg.de

Received 4 March 2014; Revised 16 May 2014; Accepted 9 June 2014; Published 13 August 2014

Academic Editor: Emanuele Boselli

Copyright (c) 2014 Daniela Küllenberg de Gaudry et al. This is an open access article distributed under the Creative Commons Attribution License, which permits unrestricted use, distribution, and reproduction in any medium, provided the original work is properly cited.

High intake of omega-3 fatty acids (n-3 FAs) from fish has shown to reduce metastatic progression of prostate cancer. This clinical trial investigated the influence of high n-3 FA intake (marine phospholipids, MPL) on the FA composition of blood lipids, lysophosphatidylcholine (LPC), and on lipoproteins in prostate cancer patients and elderly men without prostate cancer. MPL supplementation resulted in a significant increase of n-3 FAs (eicosapentaenoic and docosahexaenoic acid) in blood lipids, while arachidonic acid (n-6 FA) decreased significantly. Low density lipoprotein (LDL) and high density lipoprotein (HDL) increased significantly, but the LDL increase was observed only in subjects with an inactive tumour. Similarly, LPC plasma concentration increased significantly only in patients without tumour. The missing increase of LDL and LPC after MPL supplementation in patients with actively growing (metastasizing) prostate cancer suggests that tumour cells have an elevated demand for LDL and LPC. Due to the MPL-induced increase of n-3 FAs in these blood lipids, it can be assumed that especially actively growing and metastasizing prostate cancer cells are provided with elevated amounts of these antimetastatic n-3 FAs. A hypothetic model explaining the lower incidence of metastatic progression in prostate cancer patients with high fish consumption is presented.

\section{Introduction}

Prostate cancer (PCa) is one of the most common cancers in men over the world [1]. Dietary factors have been found to play an important role during PCa and special attention has been given to dietary fat. An important study by Augustsson et al. showed that a high fish intake ( $>3$ times per week) resulted in approximately $40 \%$ reduction of aggressive, metastatic PCa but did not influence the overall PCa incidence [2]. Similar effects were confirmed by a meta-analysis, in which a high fish consumption was demonstrated to lower the incidence of metastatic PCa by $44 \%$ and its mortality by $63 \%$ [3]. This effect has been shown to be mainly attributable to the $n-3$ fatty acids (FAs) found in fish [4].

The mechanisms by which n-3 FAs could have the potential to impede the formation of metastases are probably based on n-3FAs as precursors of eicosanoids, which are less prometastatic and less inflammatory than eicosanoids derived from n-6FAs [5]. Eicosanoids are lipid mediators, 
tissue hormones with autocrine or paracrine activity, which are involved in many physiological processes such as platelet function, immune response, and pain modulation. Dysregulation of eicosanoid biosynthesis has been linked to inflammation, infertility, allergy, degenerative diseases, atherosclerosis, ischemia, metabolic syndrome, and, most importantly, to cancer [6]. For eicosanoid biosynthesis, FAs have to be liberated from membrane phospholipids by lipolytic enzymes, which are predominantly phospholipases A2. Thereafter cyclooxygenases (COX) or lipooxygenases (LOX), which are the first step enzymes in the transformation of $n-3$ or n6 FAs, convert the previously liberated FAs into different eicosanoids. An important example is the biosynthesis of prostaglandins E (PGE) by COX. $\mathrm{PGE}_{2}$, which is derived from arachidonic acid (AA), is highly active in promoting metastatic spread and aggressive tumour growth while $\mathrm{PGE}_{3}$, derived from the n-3 FA EPA, is less active [5, 7-9]. This, and the fact that the conversion of EPA is faster than the conversion of AA by the same enzyme (COX), n-3 FAs have the potential to act antimetastatic [5]. Thus, in tumour cells which often have an increased eicosanoid biosynthesis, the n-3/n-6 FAs ratio in the cellular membranes could determine the aggressiveness of the tumour cells.

However, to change the membrane n-3/n-6 FA ratio, the cells have to be supplied with the respective essential FAs. In blood, FAs are predominantly transported by lipoproteins. From there, FAs can be supplied to the cells, either as lysophosphatidylcholine (LPC) after the action of LCAT (lecithin-cholesterol-acyltransferase) [10], as free FAs after their cleavage from other lipoproteins like LDL, VLDL, or IDL by lipases, or taken up with the whole LDL lipoprotein via its receptor [11].

As discussed above, the incidence of metastatic PCa has shown to be reduced by about $40-50 \%$ if patients ingested high amounts of n-3FAs [2]. During this clinical trial we investigated the interplay between metastatic growth of $\mathrm{PCa}$ and the effects of n-3 FA uptake on (i) blood FA composition (n-3/n-6 FA ratio), (ii) lipoprotein-, and (iii) LPC levels in patients with PCa as well as in elderly men without PCa (controls). The clinical trial was performed in the south western region of Germany, where fish consumption is usually low due to its scarcity in that area. Therefore, the study population consisted of low fish consumers. To establish FA-, lipoprotein, and LPC levels representative for high fish consumption, all subjects were supplemented with n-3 FAs given as marine phospholipids (MPL) for 3 months.

MPL is an n-3 FA-rich extract from salmon roe containing high amounts of phospholipids (PLs) containing the n-3 FAs eicosapentaenoic acid (EPA) and docosahexaenoic acid (DHA). Its composition (approximately $1 / 3$ of the $n-$ 3 FAs are bound to PLs and $2 / 3$ are bound to triglycerides) resembles the FA composition of fish better than other n3 FA supplements like fish oil, which contains no PLs [12]. It has been shown that the uptake and utilization of n3 FAs bound to PLs is more efficient than those bound to triglycerides (TGs) [13-15], but the metabolism of dietary ingested PLs has not been thoroughly investigated and many mechanisms remain to be clarified. However, it has been shown that, unlike TGs, they are almost completely absorbed as free fatty acids (FFAs) and LPC in the intestine. Following absorption, they are reesterified into PLs and incorporated mainly into chylomicrons to enter the bloodstream. Also, about $20 \%$ of the ingested PLs are absorbed passively and incorporated directly into high density lipoproteins (HDL) [16]. Additionally, other investigations with the same MPL formulation have found positive effects, for example, in the study of Taylor et al. patients with tumour associated weight loss (cachexia) achieved weight stabilization after MPL supplementation. These effects seem to be larger when compared to formulations of n-3 FA bound only to TGs. Therefore, supplementation with MPL is expected to be more effective in a relatively lower dose than fish oil or ethyl ester formulations regarding eicosanoid synthesis and its implications. In a previous study, a daily dose of $1.5 \mathrm{~g}$ MPL was shown to be enough to considerably change the n-3/n-6 FA ratio [17], while a study supplementing fish oil had similar effects, but with double the dosage (3 g n-3 FAs daily) [9].

\section{Materials and Methods}

2.1. Study Characteristics. The "Prostagen study" (registration ID: DRKS00000319 and UTN: U1111-1113-4482) was a cohort clinical trial in which patients with prostate cancer (PCa) and subjects without cancer (control group) were supplemented with marine phospholipids extract (MPLs). The clinical trial was designed based on the primary outcome of the study, which is not going to be addressed in this paper. Here, we describe the results of the trial in relation to the effects of MPL supplementation on the lipid and FA levels on the study population (secondary outcomes).

2.2. Study Population. The study population consisted of patients with prostate cancer (PCa) and subjects without PCa. All participants signed an informed written consent before study enrolment. The study protocol was approved by the "Ethics Committee of Freiburg University." Patients were identified and recruited in Freiburg in three different centres, at the department of medical oncology at the "Tumor Biology Center," the urological ward in the "Loretto Hospital" and the "Urological Practice U3" for a period of 23 months between December 2009 and October 2011.

PCa patients were included with various cancer stages, receiving different therapies, for example, surgery, radiation, hormone therapy, chemotherapy, or active surveillance. A total sample size of 150 patients (50 patients without $\mathrm{PCa}, 50$ patients with $\mathrm{PCa}$ and Gleason score $\leq 7 \mathrm{a}$, and 50 patients with $\mathrm{PCa}$ and Gleason score $\geq 7 \mathrm{~b}$ ) was calculated according to the primary endpoint of the study.

Inclusion criteria for PCa patients were male subjects older than 18 years with histologically proven PCa and who signed a written informed consent. Inclusion criterions for subjects without PCa were male subjects without PCa or any other tumour, aged 70 years or older (According to the American Cancer Society, the median age of PCa diagnosis is 66 years [18]. In order to comply with the certainty (needed for analyzing the primary endpoint of the study) that patients without PCa would not become PCa in their 
remaining lifetime, patients without PCa were to be 70 years or older. This brings selection bias into the study. However, this risk was taken, since it does not affect the primary endpoint of the study.). PCa diagnosis was defined as being excluded by the following criteria: (1) negative results from a $12 x$ prostate punch biopsy and PSA values below $10 \mathrm{ng} / \mathrm{mL}$; (2) individuals with benign prostatic hyperplasia $(\mathrm{BPH})$, who had a transurethral resection of the prostate (TUR-P) and reported histological negative results; or (3) individuals without PCa diagnosis and with PSA levels less than $4 \mathrm{ng} / \mathrm{mL}$ and no family history of prostate cancer.

Subjects were excluded from this study if they had a known allergy to seafood, malabsorption, impaired coagulation, or other severe internal diseases, psychiatric or disorders of the CNS, and subjects who were already taking supplementary n-3 FAs.

2.3. Treatment Protocol. MPL was obtained from Membramed Health Food GmbH (trade name: Vitalipin). It is a salmon roe extract consisting of $29 \%$ phosphatidylcholine and approximately $70 \%$ neutral lipids. The n-3 FA profile is $18 \%$ eicosapentaenoic acid (EPA) and 26\% docosahexaenoic acid (DHA) bound to PLs and neutral lipids [19]. It is formulated in soft gelatine capsules (500 mg/capsule), providing $223 \mathrm{mg}$ EPA and $256 \mathrm{mg}$ DHA daily (1/3 as PLs and 2/3 as TGs).

Participating subjects were asked to take one $500 \mathrm{mg}$ capsule MPL (Vitalipin) three times a day with their meals for a period of 3 months. Each subject received a patient diary in which capsule intake and fish consumption were to be documented weekly. Patients were interviewed before and after MPL intervention. During each interview subjects were surveyed for their nutritional habits (including their fish consumption), medical family history, current medication, and/or intake of supplements. Blood samples were collected for blood routine analysis, lipid electrophoresis, and plasma FA analysis.

2.4. Blood Sampling and Analysis. Blood samples were collected from each included subjects before and after MPL supplementation for determining blood parameters, FA analysis, and LPC analysis.

Blood parameters were determined after collecting EDTA blood samples $(1 \times$ S-Monovette $2.7 \mathrm{~mL}$ with $1.6 \mathrm{mg}$ EDTA/mL blood, Sarstedt, Nümbrecht, Germany) and 1 serum tube $(1 \times$ S-Monovette $9 \mathrm{~mL}$, Sarstedt, Nümbrecht, Germany) before and after MPL supplementation. The following parameters were determined: prostate specific antigen (PSA), C-reactive protein (CRP), aspartate transaminase (AST), alanine transaminase (ALT), cholinesterase (CHE), albumin, leukocytes, thrombocytes, triglycerides, total cholesterol, very low density lipoproteins (VLDL), low density lipoproteins (LDL), and high density lipoproteins (HDL), which were determined in the clinical chemistry routine laboratory according to standard procedures.

For FA analysis blood samples were collected in EDTA tubes $(1 \times$ S-Monovette $9 \mathrm{~mL}$ with $1.6 \mathrm{mg}$ EDTA $/ \mathrm{mL}$ blood, Sarstedt, Nümbrecht, Germany) and centrifuged for $10 \mathrm{~min}$ at $2000 \mathrm{rpm}$ at room temperature, the resulting plasma was stored in aliquots of $500 \mu \mathrm{L}$ at $-80^{\circ} \mathrm{C}$ until analysis.

After lipid extraction of blood plasma with the methods of Bligh and Dyer (extraction with chloroform/methanol), [20] the total lipid fraction was separated into two fractions with solid-phase extraction (SPE, over an aminopropyl column), namely, the phospholipids and the neutral lipids, according to the procedure validated and described by Taylor et al. [21]. Afterwards, FA analysis was performed with gas chromatography (GC) according to Taylor et al. [21]. GC analysis required derivatization; therefore all fractions were previously methylated with TMSH (Macherey \& Nagel, Düren, Germany). Analysis was performed with a HP 5890 Series II Plus, equipped with an Agilent Technologies (Böblingen, Germany) DB-23 column ( $30 \mathrm{~m}, 0.25 \mathrm{~mm}$ ID, $0.25 \mu \mathrm{m}$ ) with helium at $1 \mathrm{~mL} / \mathrm{min}$, oven temperature programming starting with $150^{\circ} \mathrm{C}$ for $3 \mathrm{~min}$, up to $220^{\circ} \mathrm{C}$ with a rate of $5^{\circ} \mathrm{C} / \mathrm{min}$, $220^{\circ} \mathrm{C}$ for $3.5 \mathrm{~min}$, split injection $(1: 100)$, injector temperature $260^{\circ} \mathrm{C}$, and FID at $280^{\circ} \mathrm{C}$.

LPC analysis was performed with high performance thin layer chromatography (HPTLC) after lipid extraction (as mentioned above) from blood plasma according to the method described by Taylor et al. [17]. The plasma samples and five calibration standard solutions of LPC (ranging from 80 to $400 \mu \mathrm{M}$, Sigma, Steinheim, Germany) were dissolved in $\mathrm{NaCl}$ aqueous solution and extracted with chloroform/methanol. Dry extracts (plasma and calibration standard solutions) were dissolved in hexane/isopropanol/ $\mathrm{H}_{2} \mathrm{O}$ and applied to a preconditioned HPTLC plate (Merck, Darmstadt, Germany) with the Camag Automatic Sampler TLC III. After development, plates were dried and stained with a copper sulfate/phosphoric acid solution. Quantification was performed with a Camag TLC-Scanner II equipped with a tungsten bulb at $530 \mathrm{~nm}$.

2.5. Statistics. The statistical analysis was performed with SigmaStat 3.10 (Systat Software Inc., USA, 2004) and SPSS 15.0 for Windows. Distribution was analysed with the ShapiroWilk test. Student's $t$-test and one-way analysis of variance (ANOVA) were performed if data were normally distributed; otherwise Mann-Whitney $U$ test and Kruskal-Wallis one-way ANOVA on ranks were used.

\section{Results}

The recruitment totalled 159 subjects, out of which 124 finished the study (Figure 1 and Table 1).

The results presented hereafter are based on a perprotocol analysis $(n=124)$. Most PCa patients had a transurethral resection of the prostate (TUR-P) or a radical retropubic prostatectomy (RRP) and were in remission after surgery. Subjects without PCa had mainly benign prostate hyperplasia $(\mathrm{BPH})$ and were recruited also after surgical intervention (mainly TUR-P, which confirmed the absence of $\mathrm{PCa}$ ). Since the control subjects had to be at least 70 years old to comply with the inclusion criteria, they had a slightly higher median age than patients. 


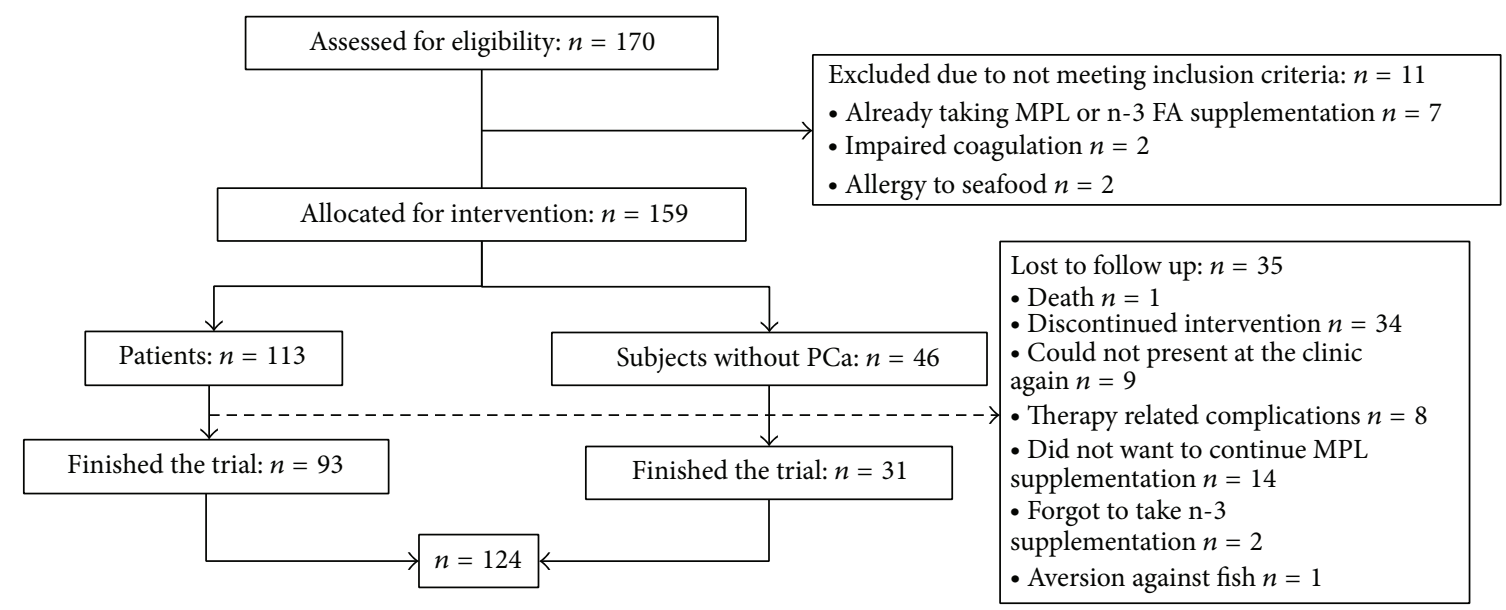

Figure 1: Flow chart of the Prostagen study.

TABLE 1: Baseline characteristics of the study population.

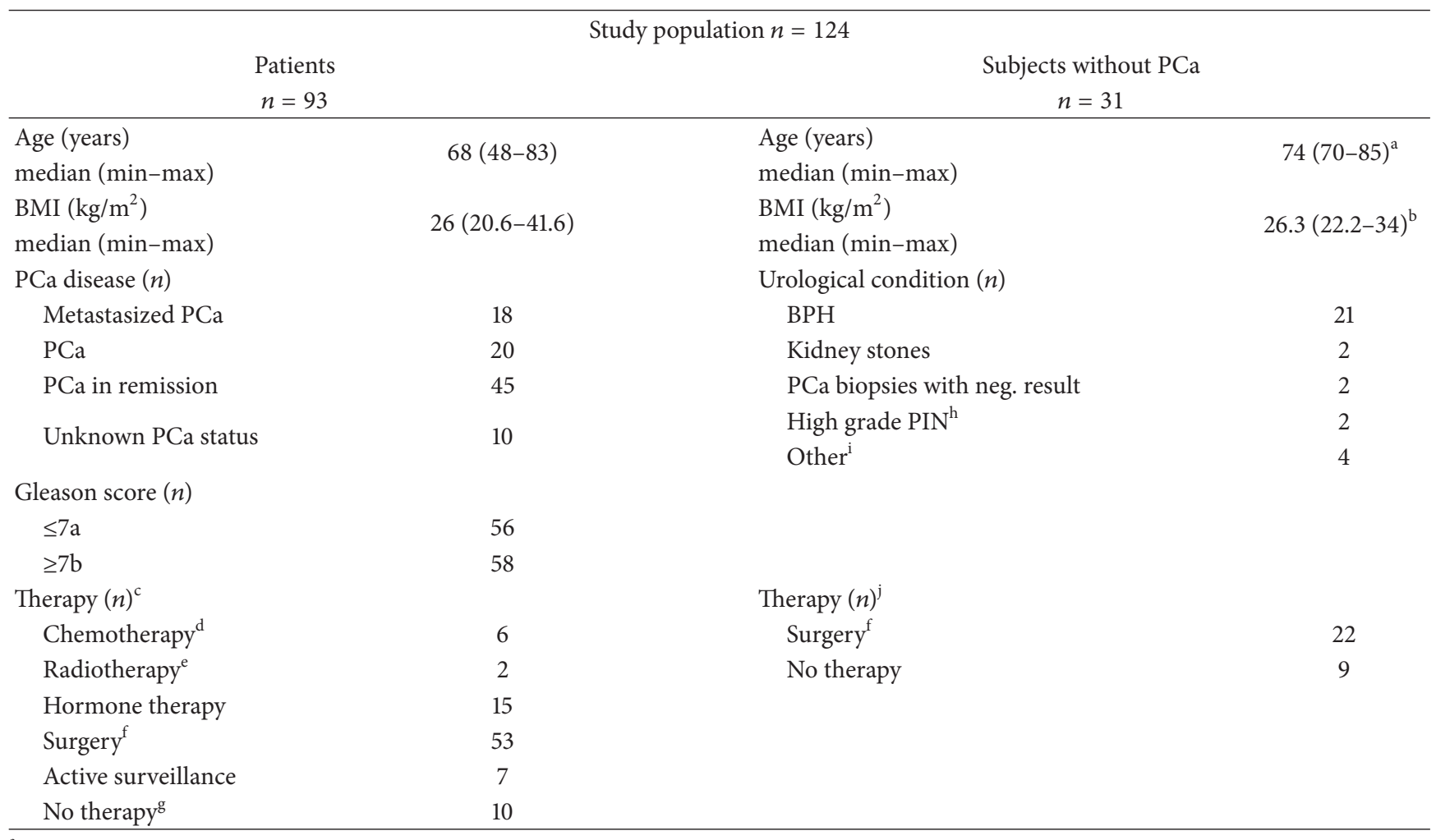

${ }^{a}$ Age difference between both groups was statistically not significant $(P=0.099)$.

${ }^{\mathrm{b}} \mathrm{BMI}$ difference between both groups was statistically not significant $(P=0.46)$.

${ }^{\mathrm{c}}$ Referred to the ongoing therapy at study enrolment.

${ }^{\mathrm{d}}$ Docetaxel, taxol, folfox, or taxotere.

${ }^{\mathrm{e}}$ Including brachytherapy.

${ }^{\mathrm{f}}$ TUR-P or RRP with or without lymphadenectomy.

${ }^{\mathrm{g}}$ No therapy in the last 6 months.

${ }^{\mathrm{h}}$ High-grade prostatic intraepithelial neoplasia.

${ }^{\mathrm{i}}$ Strangury, renal hematoma, bladder neck stenosis, and hematuria.

${ }^{j}$ TUR-P, transurethral incision of the prostate (TUIP), percutaneous nephrolithotomy, bladder neck incision, spermatocelectomy, open adenoma enucleation, transurethral laser vaporization, or placement of prostatic stent. 


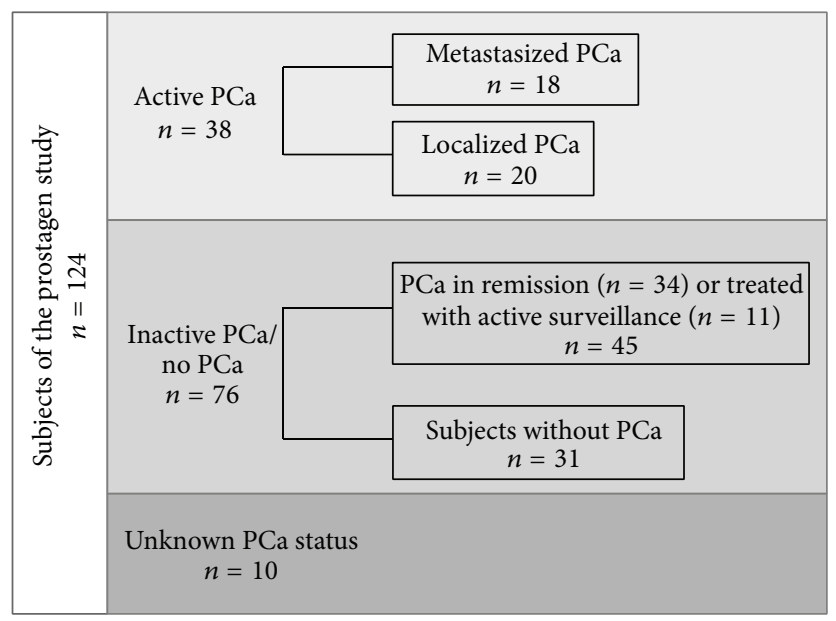

FIGURE 2: Study population grouped according to PCa diagnosis.

TABLE 2: Blood parameters in the study population before and after MPL supplementation.

\begin{tabular}{lccr}
\hline Blood parameter & Before MPL & After MPL $^{*}$ & $P$ \\
\hline Albumin $(\mathrm{g} / \mathrm{dL})$ & $4.1(2.8-5)$ & $4.3(2.6-5)$ & $<0.001$ \\
Erythrocytes $(\mathrm{Mio} / \mu \mathrm{L})$ & $4.2(2.7-5.7)$ & $4.6(2.8-5.7)$ & $<0.001$ \\
Haematocrit $(\%)$ & $38.7(22.7-50.3)$ & $41.9(24.7-50.3)$ & $<0.001$ \\
CRP $(\mathrm{mg} / \mathrm{L})$ & $12(0.7-260)$ & $2(0.7-305)$ & $<0.001$ \\
PSA $(\mu \mathrm{g} / \mathrm{L})$ & $2.3(0-1085)$ & $0.4(0-1117)$ & $<0.001$ \\
Thrombocytes $(1000 / \mu \mathrm{L})$ & $219(27-481)$ & $218(99-484)$ & n.s. \\
Leucocytes $(1000 / \mu \mathrm{L})$ & $7.2(3.2-16.4)$ & $6.3(2.8-24.1)$ & $<0.001$ \\
CHE $(\mathrm{U} / \mathrm{L})$ & $7419(2185-13950)$ & $8221(3959-12635)$ & $<0.001$ \\
AST $(\mathrm{U} / \mathrm{L})$ & $28.5(9-316)$ & $28(16-79)$ & n.s. \\
ALT $(\mathrm{U} / \mathrm{L})$ & $26(5-170)$ & $26(6-120)$ & n.s. \\
\hline
\end{tabular}

Most patients underwent surgery shortly before MPL intervention.

For the analysis of results, subjects were grouped according to their diagnosis taken from their medical records (Figure 2). The first group included patients with active PCa $(n=38)$, which were further divided into patients with metastasized PCa $(n=18)$ and localized PCa $(n=20)$. In both, metastasized and localized PCa, the tumour was growing and patients were subjected to tumour therapy. The second group of subjects was defined by patients with inactive or no PCa $(n=76)$, including patients with PCa in remission-in most cases after surgery-and patients under active surveillance or without therapy in the past 6 months $(n=45)$, and subjects without PCa $(n=31)$. The third group included subjects for whom the medical condition could not be clearly defined ( $n=10$, most of this patients did not agree to undergo further diagnosis) and were therefore not considered when analysing differences between the groups of subjects; otherwise, all subjects were included into the analysis.

3.1. MPL Effects on Blood Routine Analysis. Blood routine analysis shows significant changes in most parameters after MPL supplementation. It is important to mention that PSA values are to be evaluated with reservation, since most patients underwent surgery during the intervention. Besides the fact that many factors influence PSA values, the significant decrease observed during this study (Table 2) is probably attributed to the tumour therapy and not to MPL supplementation. Also, since most subjects had surgery a few days before study enrolment, their inflammatory markers like CRP were elevated before MPL supplementation. Its normalization (decrease) observed after the intervention should not be attributed to MPL supplementation but rather to the usual decline after surgical intervention.

3.2. Analysis of Nutritional Habits. Each subject's nutritional habits were determined before and after MPL intervention, including their regular consumption of meat, butter, fish, cheese, eggs, and vegetable oil (Quantities of each food product were grouped in $>2$ /week, $1-2 /$ week, 1-2/month, or no intake at all. Only the following fish products (due to their high n-3 FA content) were considered: salmon, herring, tuna, and mackerel.). The reported nutritional habits confirmed the assumption of the low fish intake in the south-western region of Germany. According to other studies analysing fish consumption (e.g., Augustsson et al. [2]), we defined a high fish intake as $\geq 3$ portions of fish per week. The present 
TABLE 3: Blood lipids before and after MPL supplementation.

\begin{tabular}{|c|c|c|c|}
\hline & Before MPL (mg/dL) & After MPL (mg/dL) & Change (\%) \\
\hline $\begin{array}{l}\text { Total cholesterol } \\
\text { median (min-max) }\end{array}$ & $194(80-349)$ & $221(115-362)$ & $+14 \%^{* *}$ \\
\hline $\begin{array}{l}\text { LDL cholesterol } \\
\text { median (min-max) }\end{array}$ & $121(36-277)$ & $139(42-282)$ & $+15 \%{ }^{* *}$ \\
\hline $\begin{array}{l}\text { HDL cholesterol } \\
\text { median (min-max) }\end{array}$ & $49(23-106)$ & $57(21-125)$ & $+16 \%{ }^{* *}$ \\
\hline $\begin{array}{l}\text { LDL : HDL } \\
\text { mean } \pm \text { SD }\end{array}$ & $2.8 \pm 1.3$ & $2.7 \pm 1.4$ & - \\
\hline
\end{tabular}

Analysis performed with lipid electrophoresis with nonfasting blood samples. Mean \pm SD values are shown for normally distributed data, otherwise median $(\min -\max )$ values were given.

${ }^{* *}$ Significance level $P<0.001$.

study population had an average of 1 portion of fish per week. The intake of food products was analysed in relation to the lipid and fatty acid level, without finding any significant associations.

3.3. Compliance with MPL Supplementation. The compliance with MPL supplementation was good. Ten subjects (8\%) reported fish-oil belching from time to time, which caused no discomfort, and only 11 subjects (9\%) occasionally had fish-oil taste after MPL ingestion. Other effects which might be related to MPL supplementation were fish-oil smell in urine and/or stool (2 subjects, 1.6\%), bloating and stomach discomfort ( 2 subjects, $1.6 \%$ ), reduced coagulation (Coagulation parameters were not measured during the study. Two subjects reported slightly more bleeding after injury and/or easy hematoma formation.) (2 subjects, 1.6\%), general fishoil smell (1 subject, $0.8 \%)$, and improved male potency (1 subject, $0.8 \%)$. There were no complications resulting in noncompliance of MPL supplementation.

3.4. Influence of MPL on Blood Lipids. Blood lipids were measured at baseline and after 3 months of MPL supplementation. It was not possible to draw blood samples in a fasting state since subjects were mostly recruited after having breakfast. Since triglycerides and VLDL are sensible to food intake, the values are not shown.

The study population had normal median cholesterol levels before MPL supplementation (Cholesterol reference values according to the DGFF (Lipid Liga) e.V.). Differences on the initial values of cholesterol were analysed in the groups of subjects revealing no significant differences. Subjects taking lipid lowering medication (about 38\% of subjects) had significantly lower LDL initial values than all other participants, whereas HDL remained unaffected.

After MPL supplementation, the measured total cholesterol, LDL, and HDL levels increased significantly in the whole study population about $15 \%$ (Table 3 ). The change of LDL and HDL levels was not affected if subjects were prescribed lipid lowering medication.

Differences in the lipid change between each group of subjects (Figure 2) were analysed. The results show that patients with metastasized PCa had almost no change in their total cholesterol levels after MPL supplementation, whereas all other patients and subjects without PCa had a significant increase. Detailed analysis showed that these differences were found mainly in the LDL fraction of total cholesterol (Figure 3). In regard to HDL cholesterol change, no significant differences were found between each group of subjects.

3.5. Influence of MPL on the Fatty Acid (FA) Composition in Plasma. Nine FAs in each subject before and after MPL supplementation were analysed (Table 4). Since the plasma sample of one patient could not be used for the FA analysis, the results were based only on a study population of 123 subjects.

Initial values of FAs were analysed between each group of subjects (mentioned in Figure 2). As seen in Figure 4, initial values of $\alpha$-linolenic acid (ALA) were significantly higher in patients with metastasized $\mathrm{PCa}$ in contrast to all other subjects in both the TG and PL fractions.

DHA values in the TG fraction of plasma were also significantly different between the groups of subjects. Patients with metastasized PCa had a median relative value of $0.9 \%$, whereas subjects without PCa had $0.5 \%$. No significant differences were observed for the initial values of all other FAs between the groups of subjects.

Table 4 shows the proportion of plasma FAs before and after MPL supplementation and their respective relative change. Almost all FA proportions changed significantly with MPL supplementation.

From the biologically active n-3 and n-6 FAs, EPA and DHA increased significantly after MPL supplementation in both the TG and PL fractions of plasma, whereas the AA decreased significantly. Therefore, the n-3/n-6 FA ratio improved significantly from 0.38 to 0.58 ((EPA + DHA)/AA in the TG and PL fractions, with $P<0.01$ ) (Figure 5).

Linoleic acid (LA, 18:2, n-6FA) and ALA (18:3, n$3 \mathrm{FA})$, which are precursors of the biologically FAs, EPA, and AA, were shown to increase significantly $(P<0.01)$ in both the TG and the PL fractions of plasma, after MPL supplementation.

Differences on the FA change between each group of subjects (mentioned in Figure 2) were analysed, but no significant differences were found. 

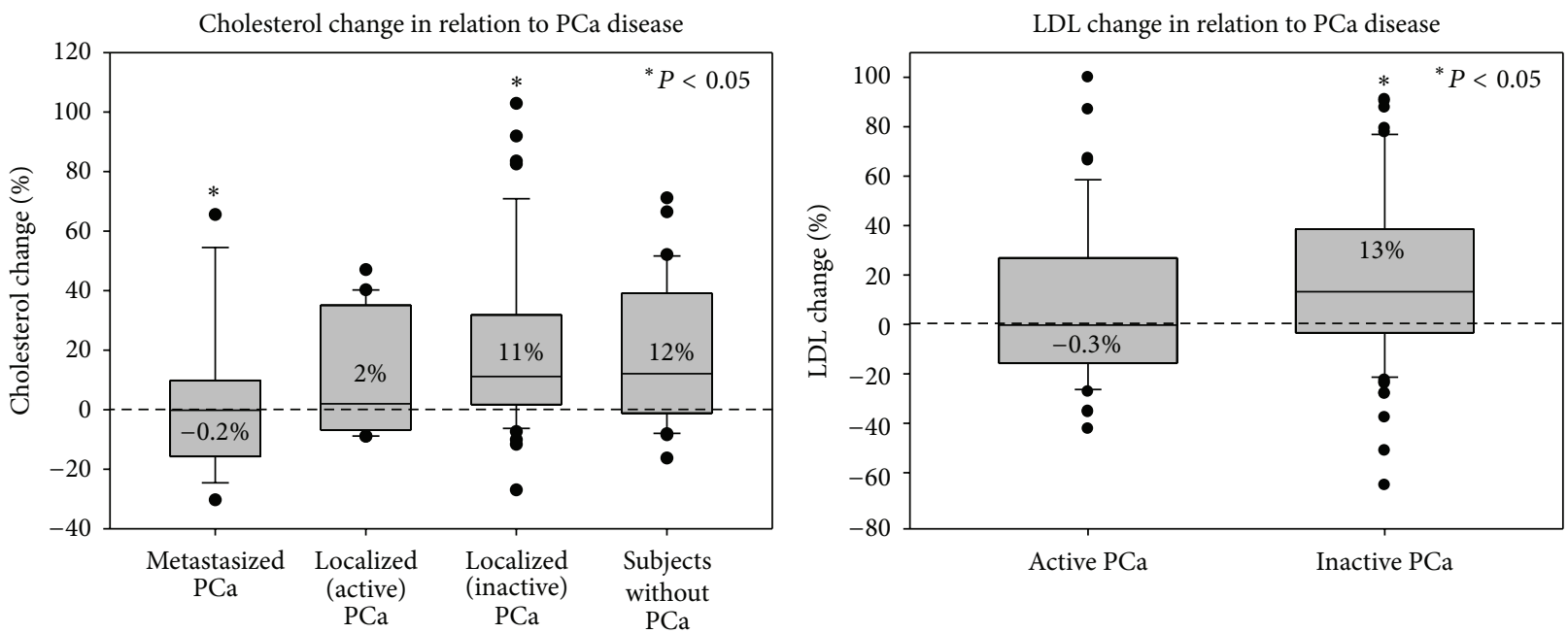

FIGURE 3: Total cholesterol and LDL change in the different groups of subjects. Left figure: Median relative change of total cholesterol after 3 months of MPL supplementation in each group of subjects. The results show a statistically significant difference $(P<0.05)$ between patients with metastasized PCa $(n=18)$ and patients with PCa in remission/active surveillance $(n=45)$. Right figure: Median relative change of LDL cholesterol after 3 months of MPL supplementation in subjects with active PCa $(n=38)$ and inactive PCa $(n=76)$. There is a statistically significant difference $(P<0.05)$ between both groups of subjects.

TABLE 4: Results of fatty acid analysis with GC.

\begin{tabular}{|c|c|c|c|c|}
\hline Fatty acid & Before MPL (\%) & After MPL (\%) & Relative change of respective $\mathrm{FA}^{\dagger}$ & $P$ \\
\hline \multicolumn{5}{|c|}{ TG-fraction } \\
\hline Myristic acid-C14:0 & $1.9(0.5-5.6)$ & $2.2(0.7-23.5)$ & $+16 \%$ & $<0.01$ \\
\hline Palmitic acid-C16:0 & $23.9(14.7-35.7)$ & $23.2(14.7-36.9)$ & $-3 \%$ & $<0.05$ \\
\hline Stearic acid-C18:0 & $3.7(1.5-8)$ & $3.7(1.9-7.2)$ & $0 \%$ & n.s. \\
\hline Oleic acid-C18:1 & $34.1(25.4-43.2)$ & $32(23.1-45.3)$ & $-6 \%$ & $<0.01$ \\
\hline Linoleic acid (LA)-C18:2 & $27.6(12.9-46.1)$ & $29.6(15.8-47.9)$ & $+7 \%$ & $<0.01$ \\
\hline$\alpha$-Linolenic acid (ALA)-C18:3 & $0.8(0.1-9.6)$ & $1(0-5)$ & $+25 \%$ & $<0.001$ \\
\hline Arachidonic acid $(\mathrm{AA})^{*}-\mathrm{C} 20: 4$ & $4.1(1.4-7.8)$ & $3.6(0.5-8.4)$ & $-12 \%$ & $<0.01$ \\
\hline Eicosapentaenoic acid (EPA)-C20:5 & $0.6(0.1-2.1)$ & $1(0.2-3.4)$ & $+67 \%$ & $<0.001$ \\
\hline Docosahexaenoic acid (DHA)-C22:6 & $0.7(0.2-2.1)$ & $1(0.1-2.1)$ & $+43 \%$ & $<0.001$ \\
\hline$\sum$ of median values & 97.4 & 97.3 & & \\
\hline \multicolumn{5}{|c|}{ PL-fraction } \\
\hline Myristic acid-C14:0 & $0.3(0.1-0.7)$ & $0.4(0.2-0.7)$ & $+33 \%$ & $<0.001$ \\
\hline Palmitic acid-C16:0 & $25.4(15.9-30)$ & $24.9(20.5-28.6)$ & $-2 \%$ & $<0.01$ \\
\hline Stearic acid-C18:0 & $11.7(8.4-16.3)$ & $12.1(8.4-15.8)$ & $+3 \%$ & $<0.001$ \\
\hline Oleic acid-C18:1 & $10.6(5.6-14.2)$ & $10.7(7.4-15.6)$ & $0 \%$ & n.s. \\
\hline Linoleic acid (LA)-C18:2 & $17.4(11.2-24.3)$ & $18.5(8.3-23.9)$ & $+6 \%$ & $<0.01$ \\
\hline$\alpha$-linolenic acid (ALA)-C18:3 & $0.2(0-3.4)$ & $0.3(0-0.9)$ & $+50 \%$ & $<0.001$ \\
\hline Arachidonic acid $(\mathrm{AA})^{*}-\mathrm{C} 20: 4$ & $9.3(3.2-14.8)$ & $8.5(4.3-14.4)$ & $-9 \%$ & $<0.001$ \\
\hline Eicosapentaenoic acid (EPA)-C20:5 & $0.8(0.2-3.4)$ & $1.4(0.5-4.1)$ & $+75 \%$ & $<0.001$ \\
\hline Docosahexaenoic acid (DHA)-C22:6 & $3(0.9-6.1)$ & $3.6(1.5-5.9)$ & $+20 \%$ & $<0.001$ \\
\hline$\sum$ sum of median values & 78.7 & 75.4 & & \\
\hline
\end{tabular}

*Normal distribution.

${ }^{\dagger}$ Change of FAs after MPL supplementation based on the median initial values.

Median relative values of each FA before and after MPL supplementation in the study population $(n=123)$. Since most of the data is not normally distributed, all values are given in median (min-max). Since C:17 lysophosphatidylcholine was used as internal standard, the results of C:17 are not shown in the table. C17 stays mostly in the PL fraction; therefore the values of the shown FAs (\% of each FA) are not based on a 100\%. 
ALA initial values in the TG fraction of plasma in relation to PCa disease

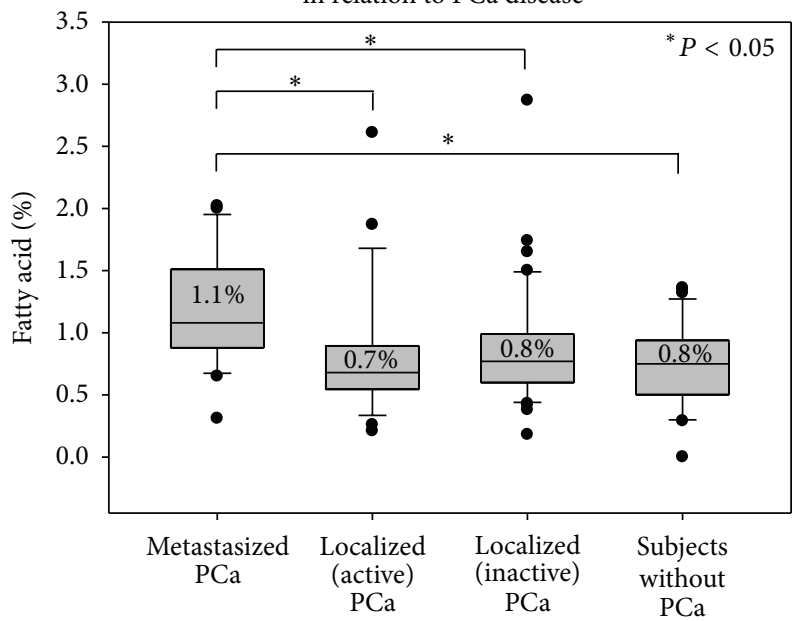

ALA initial values in the PL fraction of plasma in relation to PCa disease

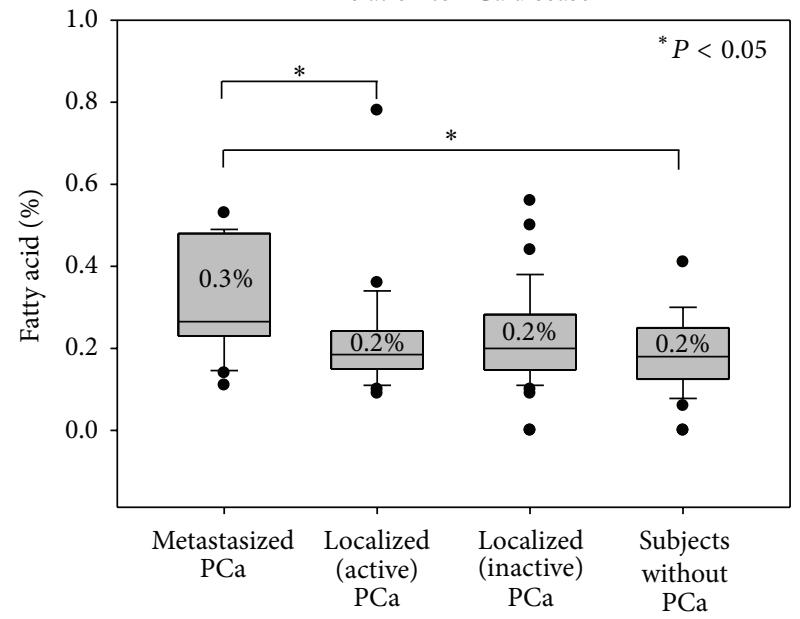

FIgURE 4: Initial values of ALA in the TG and PL fractionsbetween the different groups of subjects. Median relative initial values of ALA in the TG and PL fractions of blood plasma. Significance was tested between each group of subjects. Significant differences $(P<0.05)$ were observed between the highlighted $(*)$ groups of subjects.
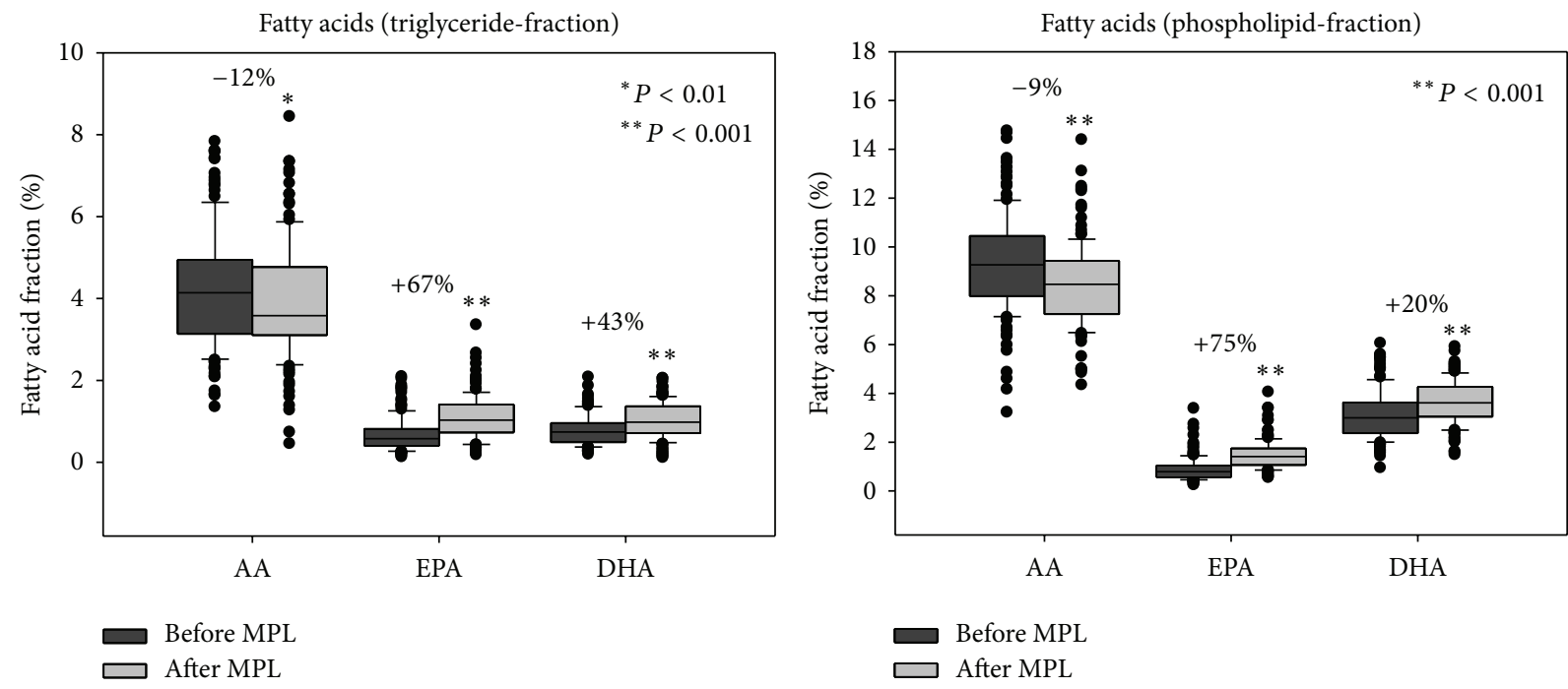

FIGURE 5: Long chain n-3 and n-6 FAs before and after MPL supplementation in the TG and PL fractions of plasma. Median relative values of the biologically active FAs AA, EPA, and DHA in the TG and PL fractions of plasma before and after 3 months of MPL supplementation in the study population $(n=123)$. The AA (n-6) decreased significantly after supplementation, whereas EPA and DHA increased significantly in both fractions. Although AA in the TG fraction and DHA in the PL fraction had a normal distribution all FAs are represented in median values for comparison.

3.6. Influence of MPL on Lysophosphatidylcholine (LPC) in Plasma. Initial values of LPC were not significantly different between the groups of subjects and had a median value of $181 \mu \mathrm{M}$. After MPL supplementation LPC increased in the whole study population to $223 \mu \mathrm{M}(P<0.001)$. When observing the LPC change in the different groups of subjects, patients with metastasized PCa had a LPC decrease after MPL supplementation, while in all other groups LPC increased. The difference between the group of patients with metastasized PCa and all others was significant $(P<0.05)$.

\section{Discussion}

During this clinical trial we found that simulating a high fish consumption in subjects with a usually rather low fish consumption by supplying MPL improved the n-3/n-6 FA ratio significantly. This was observed in all study groups without differences. MPL supplementation showed to have a good compliance compared to fish oil supplementation described in other clinical trials. For example, the study of Bruera et al., in which fish oil was supplemented to patients 
in an advanced cancer stage, showed to cause multiple gastrointestinal problems with an even lower dose [22].

Besides the MPL-induced increase of the n-3/n-6 FA ratio in all study groups, significant differences on the LDLand lysophosphatidylcholine (LPC) - increase between the groups of subjects were observed. Patients with metastasized PCa/active PCa did not show the LDL and LPC accumulation, which was present in all other groups, probably due to a higher demand of the metastasizing tumour cells for these lipids. Another significant difference between both groups of subjects was found in the initial values of ALA, possibly due to a higher eicosanoid biosynthesis from n-6 FA.

To our knowledge until today, there is no evidence showing that actively growing PCa cells have a higher LDL uptake, but there are clues that LDL plays a role in the development of PCa. An in vitro study showed that the growth inhibiting effect of simvastatin on prostate cancer cell lines was prevented when adding LDL [23]. The mechanisms by which LDL could be contributing to PCa development remain unclear, but it might be possible that LDL receptors are overexpressed in tumour cells, facilitating its uptake to comply with their lipid demand for proliferation. For example, Gilardoni et al. observed in rats that the expression of LDL-Receptor-related Protein-1 (LRP-1), which is one LDL receptor type, was higher in premalignant lesions compared to normal cells [24]. In general, a higher cholesterol demand by tumour cells has been linked to cancer progression through, for example, increased cellular proliferation, inflammation as well as regulating lipid rafts and thereby affecting signalling pathways of apoptosis. In consequence, high circulating cholesterol levels were associated with an increased risk of aggressive PCa [25]. Hence, our results are in line with studies that showed reduced cholesterol levels in men developing cancer [26-28], since tumour cells could be using cholesterol for their survival.

The probable higher LPC uptake by metastatic tumour cells observed during our study corresponds with in vitro experiments showing that metastatic tumour cells rapidly catabolize LPC as well as incorporate it into their cellular membranes, thereby changing its membrane FA composition [29]. Also, Raynor showed that PCa cell lines (PC3, DU145, and LNCaP) had a higher LPC incorporation and metabolism than normal cells [30]. During clinical trials, it was found that LPC levels were decreased in patients with an advanced cancer disease [17] and in patients with colorectal cancer [31]. Thus, the probable high LPC demand of aggressively growing tumour cells would explain the missing LPC increase observed in the group of patients with metastasized PCa. However, it would have been consistent if LPC values before giving MPL were also lower in metastasized PCa patients when compared to all other subjects, but no differences were observed before intervention. An explanation could be that all subjects had already low LPC values $(181 \mu \mathrm{M}$, normal values range from 200 to $400 \mu \mathrm{M}$ [32-35]).

It has been shown that eicosanoid synthesis from PUFAs is increased in tumour cells [36] and that the relative proportion of n-3 and n-6 PUFAs in the cellular membranes is one factor which determines the types of eicosanoids that are generated [5]. The n-6 FA AA has been described to be implicated in the development of cancer and also of metastases through the synthesis of series 2 eicosanoids, especially of $\mathrm{PGE}_{2}[5,6,37]$. With the results of this study we speculate that during metastasis an increased conversion of AA to eicosanoids takes place, since the values of $\alpha$ linolenic acid (ALA) before MPL supplementation were higher in patients with metastasized PCa compared to all other subjects. This finding supports the assumption that metastasized/active tumour cells "consume" more n-6 FAs than n-3 FAs, resulting in accumulation of the n-3-FA ALA, which is the precursor of the n-3 FA EPA.

In concordance with this finding, lower initial values of the n-6FA linoleic acid (LA, precursor of AA) would have been expected in patients with metastasized $\mathrm{PCa}$ as a consequence of a higher AA demand. While in other studies reduced proportions of LA have been found in cancer patients in comparison to healthy individuals [21] in this study no differences could be observed between the groups of patients. One explanation might be that due to the usually high AA intake in the western diet of all study participants, an increased conversion of LA to AA might not be required to fulfil the needs of the metastatic tumour cells. In addition, LA blood concentration is much higher than that of ALA (LA/ALA in PL is approximately 80, in TG approximately 34) and, thus, a possible small reduction of LA in relation to its high values cannot be clearly observed.

Based on our results, a hypothesis is proposed (Figure 7) which helps to explain the prevention of metastatic tumour growth in more than $40 \%$ of PCa patients that have high fish consumption, which was shown in different clinical trials $[2$, 3].

From our data we assume that aggressively growing PCa cells developed the ability to take up high amounts of LDL and that during metastatic transformation those cells developed the additional property to take up and metabolize high amounts of LPC (Figure 6). Thus, LDL and LPC are expected to especially supply metastatic cancer cells with high amounts of FAs.

Depending on which kind of FAs are predominantly contained in the diet ("western diet:" rich in n-6 FAs versus fish diet (or MPL): rich in n-3FAs), the FA composition of LDL and LPC will be modulated accordingly. Therefore, we assume that the diet influences the n-3/n-6 FA ratio especially in metastatic tumour cells, influencing thereby whether cells synthesize either pro- or antimetastatic eicosanoids. LDL and LPC could be regarded as Trojan horses, which become only dangerous to the metastasizing tumour cells if they were previously enriched with n-3 FAs. Patients, who respond to either western diet or fish diet with an increase in LDL and LPC, containing the respective pro- or antimetastatic FA, are therefore considered as "omega-sensitive."

\section{Conclusions}

Taken together MPL supplementation was shown to be effective in increasing the n-3/n-6 FA ratio significantly, with a very good compliance at the same time. With our results we could assume that active/metastatic tumour cells have 


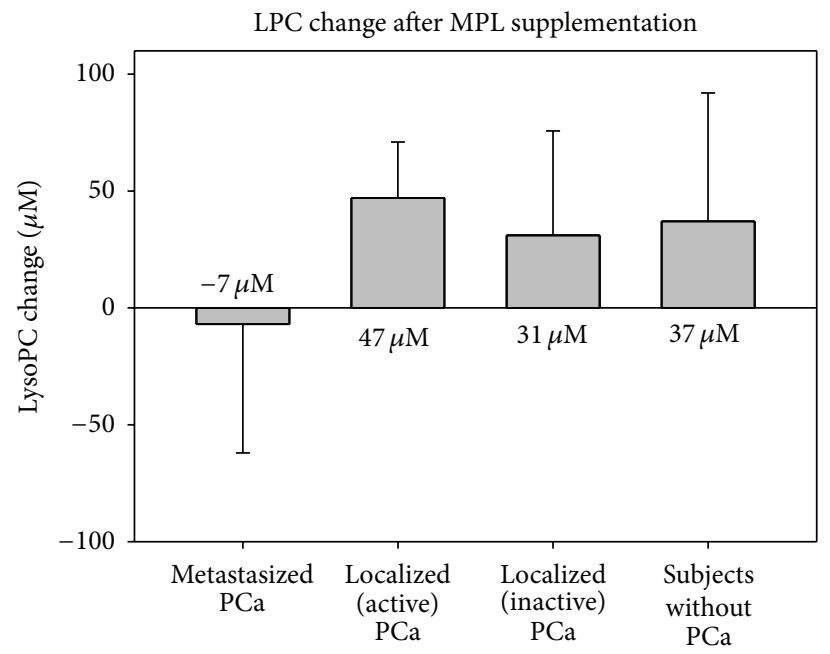

FIGURE 6: LPC change in the different groups of subjects.

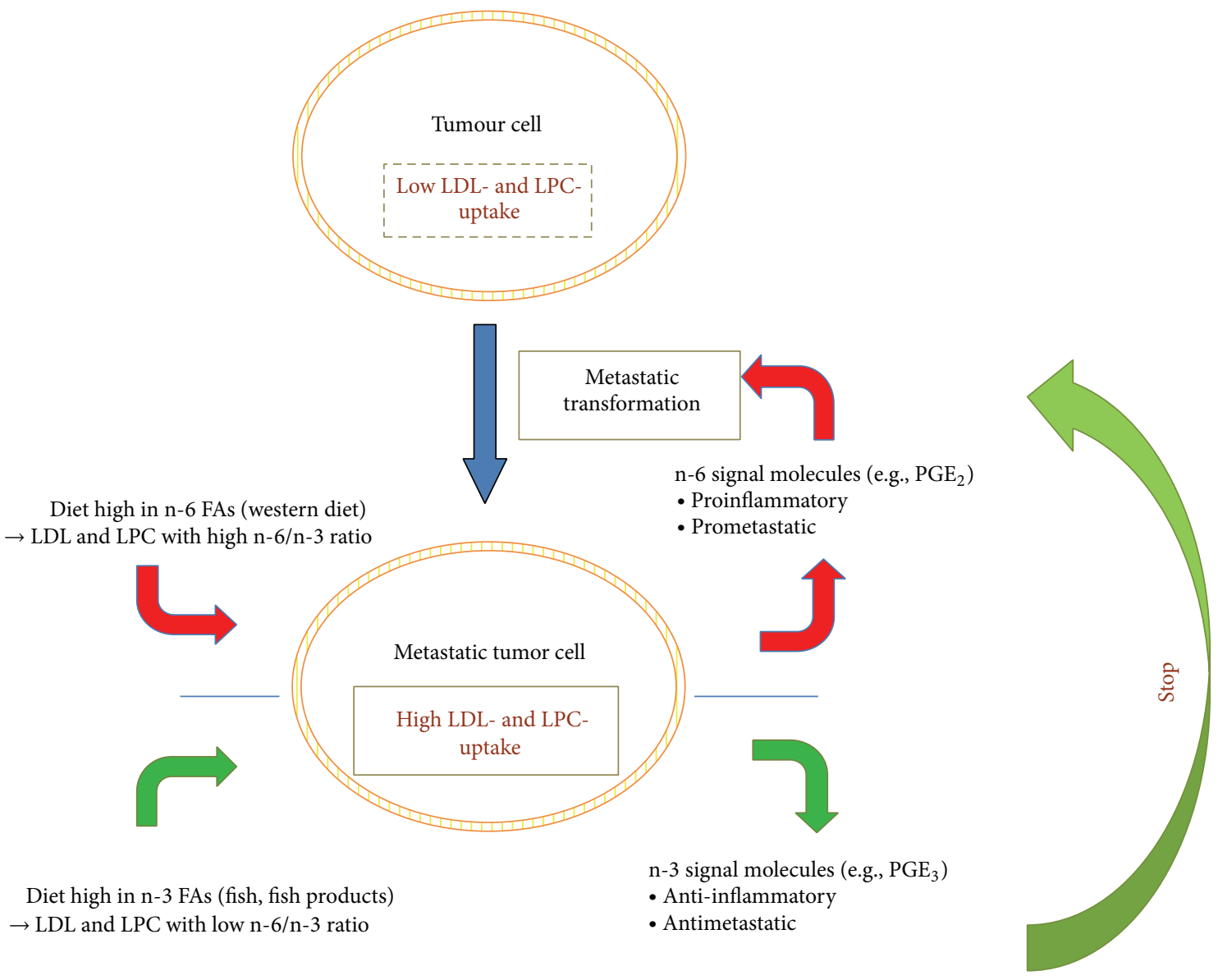

Figure 7: Hypothetic model explaining the "omega-sensitivity" found in about half of the patients with metastatic PCa.

an influence on the LDL and LPC levels, probably due to a higher demand of these lipids. Influencing the FA composition of LDL and LPC towards a higher n-3/n-6 FA ratio could convert LDL and LPC particles into Trojan horses, having the potential to influence the n-3/n-6FA ratio of tumour cells and thereby reduce the biosynthesis of proinflammatory eicosanoids. If the results of this clinical trial could be confirmed with further investigations, a high fish intake is recommended to PCa patients, since it was shown that $40-$ $50 \%$ of patients profit from this diet to impede metastatic 
development [3]. If achieving high fish consumption is not possible, MPL showed to be an efficient alternative in delivering $n-3$ FAs. These results warrant further studies to confirm the effects of an n-3 FA increase in relation to $\mathrm{PCa}$ development.

\section{Disclosure}

The Tumor Biology Center Freiburg is owner of a patent pertaining to the use of marine phospholipids for palliative cancer treatment. One of the authors, Ulrich Massing, is the inventor of the mentioned patent. All other authors declare to have no financial interests related to the publication of this work.

\section{Conflict of Interests}

The authors declare that there is no conflict of interests regarding the publishing of this paper.

\section{Acknowledgment}

Financial support was given by Federal Ministry of Education and Research Germany (Bundesministerium für Bildung und Forschung, BMBF; Project no. 0315477B).

\section{References}

[1] P. D. Baade, D. R. Youlden, and L. J. Krnjacki, "International epidemiology of prostate cancer: geographical distribution and secular trends," Molecular Nutrition and Food Research, vol. 53, no. 2, pp. 171-184, 2009.

[2] K. Augustsson, D. S. Michaud, E. B. Rimm et al., "A prospective study of intake of fish and marine fatty acids and prostate cancer," Cancer Epidemiology Biomarkers and Prevention, vol. 12, no. 1, pp. 64-67, 2003.

[3] K. M. Szymanski, D. C. Wheeler, and L. A. Mucci, "Fish consumption and prostate cancer risk: a review and metaanalysis," The American Journal of Clinical Nutrition, vol. 92, no. 5, pp. 1223-1233, 2010.

[4] M. D. Brown, C. A. Hart, E. Gazi, S. Bagley, and N. W. Clarke, "Promotion of prostatic metastatic migration towards human bone marrow stoma by Omega 6 and its inhibition by Omega 3 PUFAs," British Journal of Cancer, vol. 94, no. 6, pp. 842-853, 2006.

[5] S. C. Larsson, M. Kumlin, M. Ingelman-Sundberg, and A. Wolk, "Dietary long-chain n-3 fatty acids for the prevention of cancer: a review of potential mechanisms," The American Journal of Clinical Nutrition, vol. 79, no. 6, pp. 935-945, 2004.

[6] M. Murakami, "Lipid mediators in life science," Experimental Animals, vol. 60, no. 1, pp. 7-20, 2011.

[7] P. Sinha, V. K. Clements, A. M. Fulton, and S. OstrandRosenberg, "Prostaglandin E2 promotes tumor progression by inducing myeloid-derived suppressor cells," Cancer Research, vol. 67, no. 9, pp. 4507-4513, 2007.

[8] G. Calviello and S. Serini, Dietary Omega-3 Polyunsaturated Fatty Acids and Cancer, Springer, 2010.

[9] W. J. Aronson, J. A. Glaspy, S. T. Reddy, D. Reese, D. Heber, and D. Bagga, "Modulation of omega-3/omega-6 polyunsaturated ratios with dietary fish oils in men with prostate cancer," Urology, vol. 58, no. 2, pp. 283-288, 2001.

[10] M. Gauster, G. Rechberger, A. Sovic et al., "Endothelial lipase releases saturated and unsaturated fatty acids of high density lipoprotein phosphatidylcholine," Journal of Lipid Research, vol. 46, no. 7, pp. 1517-1525, 2005.

[11] J. L. Goldstein, M. S. Brown, R. G. W. Anderson, D. W. Russell, and W. J. Schneider, "Receptor-mediated endocytosis: concepts emerging from the LDL receptor system," Annual Review of Cell Biology, vol. 1, pp. 1-39, 1985.

[12] J. B. Luten, Seafood Research from Fish to Dish: Quality, Safety and Processing of Wild and Farmed Fish, Wageningen Academic Publishers, 2006.

[13] V. Wijendran, M. C. Huang, G. Y. Diau, G. Boehm, P. W. Nathanielsz, and J. T. Brenna, "Efficacy of dietary arachidonic acid provided as triglyceride or phospholipid as substrates for brain arachidonic acid accretion in baboon neonates," Pediatric Research, vol. 51, no. 3, pp. 265-272, 2002.

[14] B. Goustard-Langelier, P. Guesnet, G. Durand, J. M. Antoine, and J. M. Alessandri, "n-3 and n- 6 Fatty acid enrichment by dietary fish oil and phospholipid sources in brain cortical areas and nonneural tissues of formula-fed piglets," Lipids, vol. 34, no. 1, pp. 5-16, 1999.

[15] L. Burri, N. Hoem, S. Banni, and K. Berge, "Marine Omega-3 phospholipids: Metabolism and biological activities," International Journal of Molecular Sciences, vol. 13, no. 11, pp. 1540115419, 2012.

[16] O. Zierenberg and S. M. Grundy, "Intestinal absorption of polyenephosphatidylcholine in man," Journal of Lipid Research, vol. 23, no. 8, pp. 1136-1142, 1982.

[17] L. A. Taylor, L. Pletschen, J. Arends, C. Unger, and U. Massing, "Marine phospholipids-a promising new dietary approach to tumor-associated weight loss," Supportive Care in Cancer, vol. 18, no. 2, pp. 159-170, 2010.

[18] American Cancer Society, "Information and Resources for Cancer: Breast, Colon, Lung, Prostate, Skin,” http://www.cancer. org/.

[19] D. Küllenberg, L. A. Taylor, M. Schneider, and U. Massing, "Health effects of dietary phospholipids," Lipids in Health and Disease, vol. 11, article 3, 2012.

[20] E. G. Bligh and W. J. Dyer, "A rapid method of total lipid extraction and purification," Canadian Journal of Biochemistry and Physiology, vol. 37, no. 8, pp. 911-917, 1959.

[21] L. A. Taylor, V. Ziroli, and U. Massing, "Analysis of fatty acid profile in plasma phospholipids by solid-phase extraction in combination with GC," European Journal of Lipid Science and Technology, vol. 111, no. 9, pp. 912-919, 2009.

[22] E. Bruera, F. Strasser, J. L. Palmer et al., "Effect of fish oil on appetite and other symptoms in patients with advanced cancer and anorexia/cachexia: a double-blind, placebo-controlled study," Journal of Clinical Oncology, vol. 21, no. 1, pp. 129-134, 2003.

[23] T. J. Murtola, H. Syvälä, P. Pennanen et al., "Comparative effects of high and low-dose simvastatin on prostate epithelial cells: the role of LDL," European Journal of Pharmacology, vol. 673, no. 13, pp. 96-100, 2011.

[24] M. B. Gilardoni, D. G. Ceschin, M. M. Sahores, M. Oviedo, R. C. Gehrau, and G. A. Chiabrando, "Decreased expression of the low-density lipoprotein receptor-related protein-1 (LRP1) in rats with prostate cancer," Journal of Histochemistry \& Cytochemistry, vol. 51, no. 12, pp. 1575-1580, 2003. 
[25] K. Pelton, M. R. Freeman, and K. R. Solomon, "Cholesterol and prostate cancer," Current Opinion in Pharmacology, vol. 12, no. 6, pp. 751-759, 2012.

[26] R. W. Sherwin, D. N. Wentworth, J. A. Cutler, S. B. Hulley, L. H. Kuller, and J. Stamler, "Serum cholesterol levels and cancer mortality in 361662 men screened for the Multiple Risk Factor Intervention Trial," The Journal of the American Medical Association, vol. 257, no. 7, pp. 943-948, 1987.

[27] A. Keys, C. Aravanis, H. Blackburn et al., "Serum cholesterol and cancer mortality in the seven countries study," The American Journal of Epidemiology, vol. 121, no. 6, pp. 870-883, 1985.

[28] K. R. Solomon and M. R. Freeman, "The complex interplay between cholesterol and prostate malignancy," Urologic Clinics of North America, vol. 38, no. 3, pp. 243-259, 2011.

[29] P. Jantscheff, M. Schlesinger, J. Fritzsche et al., "Lysophosphatidylcholine pretreatment reduces VLA-4 and P-Selectinmediated b16.f10 melanoma cell adhesion in vitro and inhibits metastasis-like lung invasion in vivo," Molecular Cancer Therapeutics, vol. 10, pp. 186-197, 2011.

[30] A. Raynor, 2013.

[31] Z. Zhao, Y. Xiao, P. Elson et al., "Plasma lysophosphatidylcholine levels: potential biomarkers for colorectal cancer," Journal of Clinical Oncology, vol. 25, no. 19, pp. 2696-2701, 2007.

[32] K. Raffelt, D. Moka, F. Süllentrop, M. Dietlein, J. Hahn, and H. Schicha, "Systemic alterations in phospholipid concentrations of blood plasma in patients with thyroid carcinoma: an invitro ${ }^{31} \mathrm{P}$ high-resolution NMR study," NMR in Biomedicine, vol. 13, no. 1, pp. 8-13, 2000.

[33] A. Takatera, A. Takeuchi, K. Saiki, T. Morisawa, N. Yokoyama, and M. Matsuo, "Quantification of lysophosphatidylcholines and phosphatidylcholines using liquid chromatographytandem mass spectrometry in neonatal serum," Journal of Chromatography B: Analytical Technologies in the Biomedical and Life Sciences, vol. 838, no. 1, pp. 31-36, 2006.

[34] M. Kuliszkiewicz-Janus and S. Baczyński, "Application of 31P NMR spectroscopy to monitor chemotherapy-associated changes of serum phospholipids in patients with malignant lymphomas," Magnetic Resonance in Medicine, vol. 35, no. 4, pp. 449-456, 1996.

[35] M. Kuliszkiewicz-Janus, M. A. Tuz, and S. Baczyński, "Application of ${ }^{31} \mathrm{P}$ MRS to the analysis of phospholipid changes in plasma of patients with acute leukemia," Biochimica et Biophysica Acta: Molecular and Cell Biology of Lipids, vol. 1737, no. 1, pp. 11-15, 2005.

[36] D. Nie, M. Che, D. Grignon, K. Tang, and K. V. Honn, "Role of eicosanoids in prostate cancer progression," Cancer and Metastasis Reviews, vol. 20, no. 3-4, pp. 195-206, 2001.

[37] I. M. Berquin, I. J. Edwards, and Y. Q. Chen, "Multi-targeted therapy of cancer by omega-3 fatty acids," Cancer Letters, vol. 269, no. 2, pp. 363-377, 2008. 


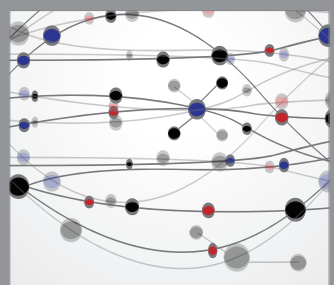

The Scientific World Journal
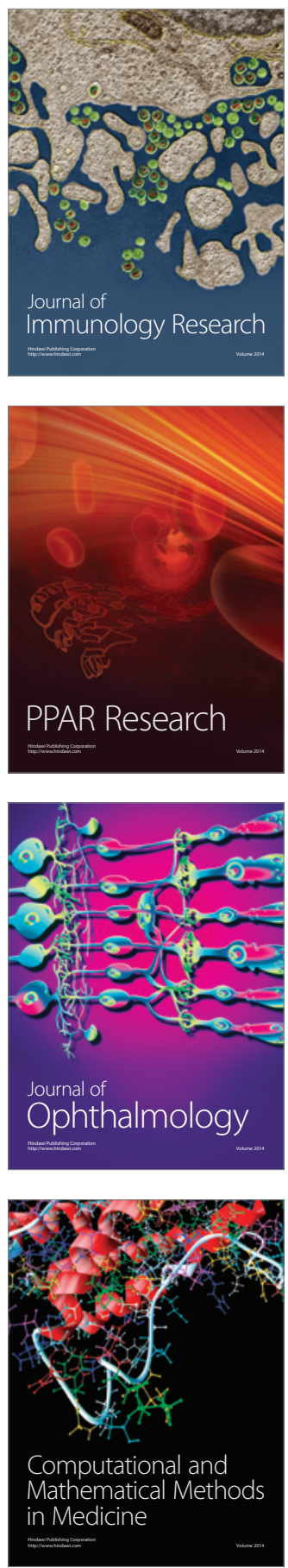

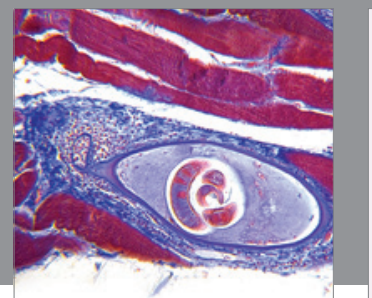

Gastroenterology

Research and Practice
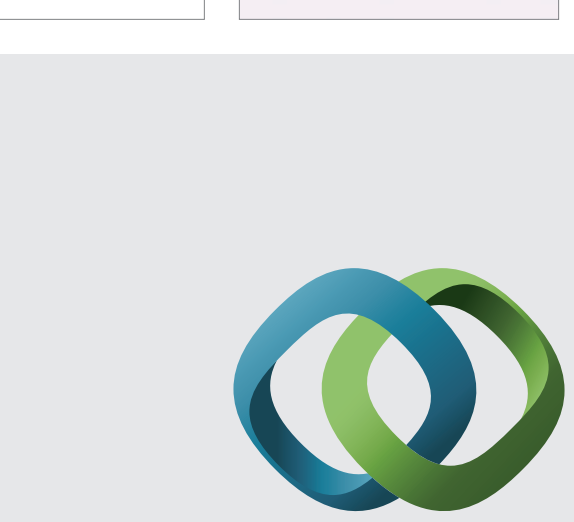

\section{Hindawi}

Submit your manuscripts at

http://www.hindawi.com
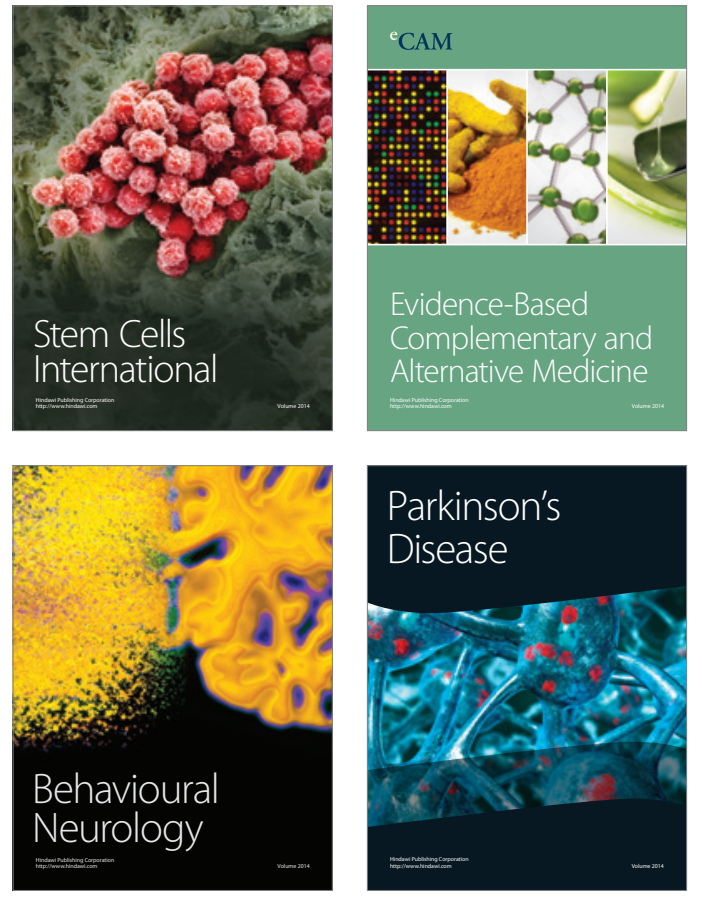
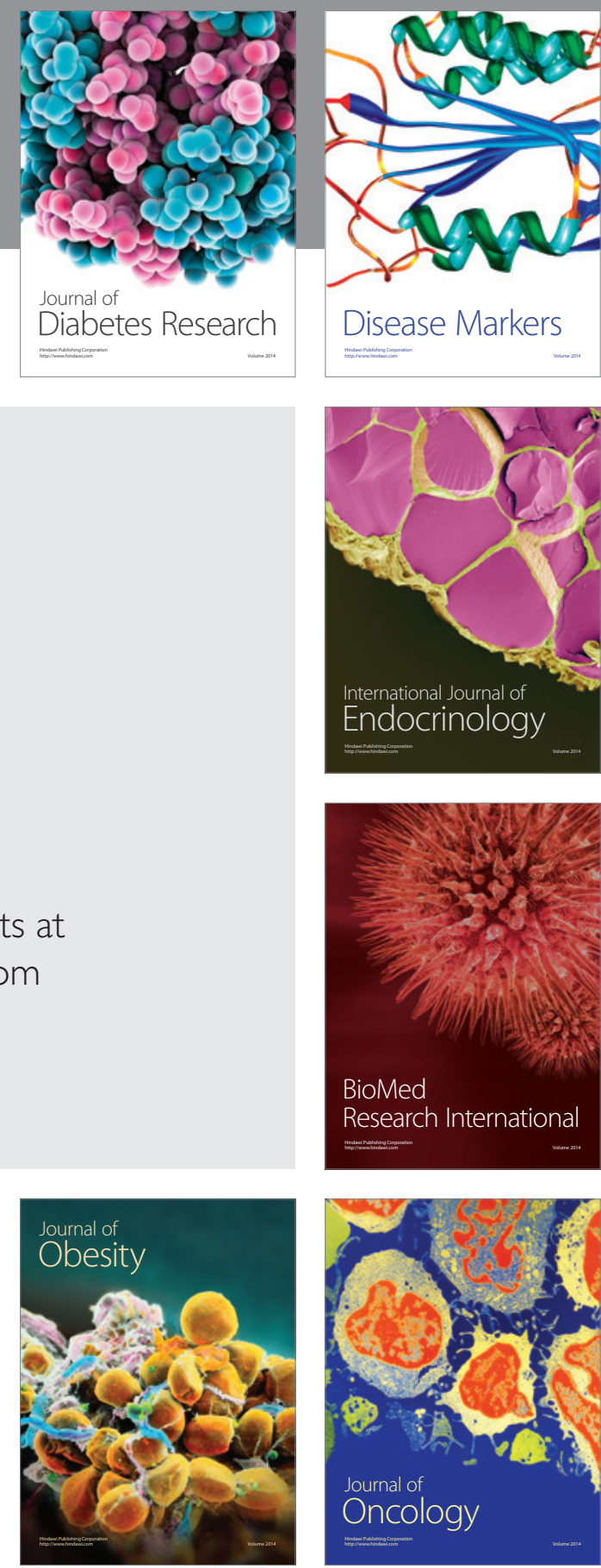

Disease Markers
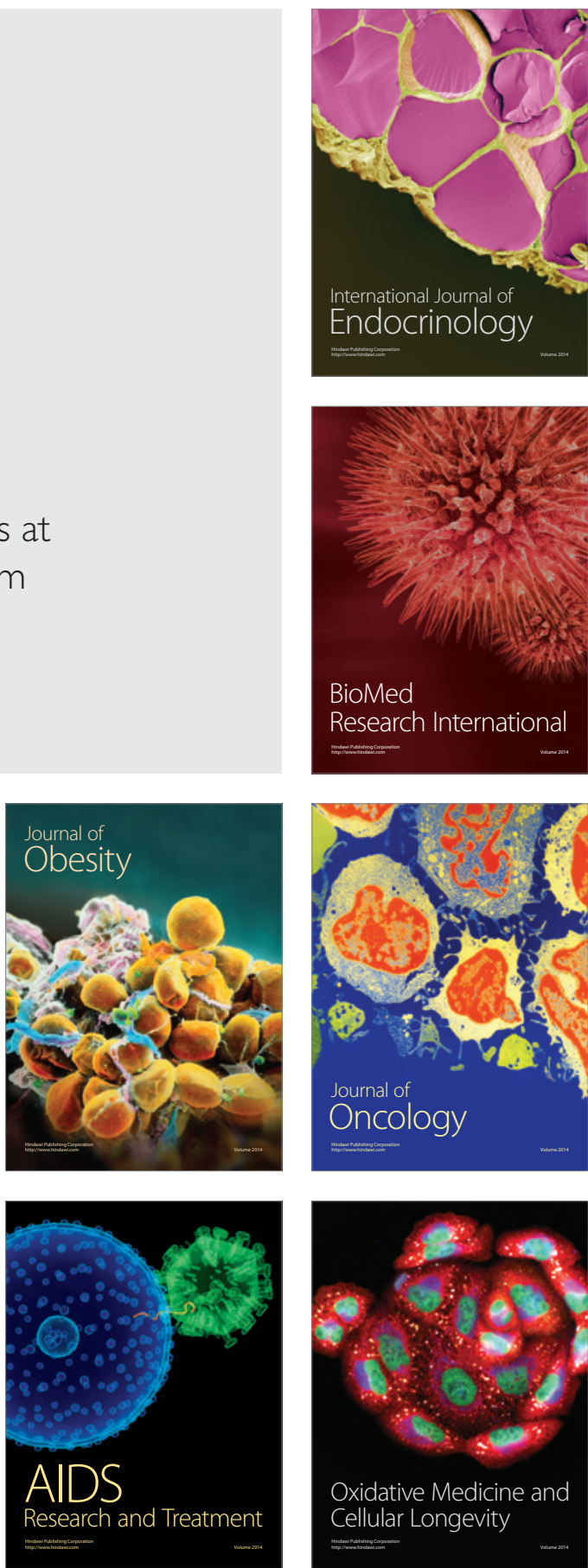